\title{
An Unusual Presentation of Tuberculosis of the Nasopharynx
}

\author{
X. Y. Yeoh", K. C. Pua \\ Department of Otorhinolaryngology, Penang General Hospital, Penang, Malaysia \\ Email: ${ }^{*}$ xy.yeoh@gmail.com
}

Received 18 April 2015; accepted 23 June 2015; published 30 June 2015

Copyright @ 2015 by authors and Scientific Research Publishing Inc.

This work is licensed under the Creative Commons Attribution International License (CC BY).

http://creativecommons.org/licenses/by/4.0/

c) (i) Open Access

\begin{abstract}
Background: Tuberculosis (TB) of the nose and nasopharynx are rare localizations for tuberculosis to manifest. Aim: We hope to present a case of tuberculosis of the nasopharynx and its rare presentation. Case presentation: This patient presented with a long standing history of headache and neck pain prior to being seen. Imaging showed a mass at the nasopharynx and a biopsy confirmed tuberculosis. Patient was initiated on anti-tuberculosis therapy with improvement in her symptoms on follow up. Conclusion: A high index of suspicion is imperative for early diagnosis.
\end{abstract}

\section{Keywords}

Tuberculosis, Nasopharynx, Cervical Spine, Pregnancy

\section{Introduction}

Tuberculosis remains a major health problem in the developing world, and the number in Malaysia continues to rise unabated, leading to high rates of morbidity and mortality [1]. While pulmonary tuberculosis is the commonest form, extra pulmonary tuberculosis still poses a threat (15\%). The diagnosis of extra pulmonary tuberculosis is challenging as it has a lower bacterial load compared to pulmonary tuberculosis and sample collection is problematic [1]. Tuberculosis of the nasopharynx is extremely rare even in countries with a high incidence of pulmonary disease. Therefore, awareness regarding its different presentations and a high index of suspicion are important for correct early diagnosis and proper initiation of treatment. We share a case who presented with headache and neck stiffness for a prolonged period of time prior to being seen. Imaging facilitated ease in obtaining a biopsy and treatment was initiated after confirmation of diagnosis of TB.

\section{Case Report}

A 32-year-old Malay woman presented with left-sided occipital headache and left-sided neck pain for 2 years, *Corresponding author. 
which started during her pregnancy, and lasted for 2 years. She only sought treatment post-partum and was given painkillers, but her headache was unresponsive to medications. She also complained of restricted movement and stiffness of the neck, but had no ear, nose, throat symptoms, chronic cough or constitutional symptoms. On examination there was slight torticollis to the left, and her range of movement of the neck was limited. There was a vague diffuse fullness over the left neck. The cranial nerves were normal. She was referred to the Ear Nose and Throat (ENT) department after imaging studies. Her chest X-ray and sputum examinations did not reveal abnormalities. Rigid nasoendoscopy revealed a mass at the left postnasal space (PNS) and a biopsy was taken (Figure 1). Computed tomography scan revealed bony destruction with enhancing soft tissue component of the left lateral mass of C1, dens of C2, left occipital condyle and clivus (Figure 2).

Biopsy of PNS mass reported caseating granulomatous lesion, consistent with mycobacterial infection. ZiehlNeelson stain for acid-fast bacilli was positive. Patient was started on anti-tuberculosis therapy. She was seen after 5 months of treatment and her endoscopy findings did not reveal any nasopharyngeal mass (Figure 1). However, a Magnetic Resonant Imaging (MRI) after 6 months of anti-tuberculosis therapy revealed persistent but smaller C2 enhancing mass (Figure 3). She was extended on anti-tuberculosis therapy for 9 months. She demonstrated symptom improvement and is still on anti-tuberculosis therapy and under follow-up to date.

\section{Discussion}

Mycobacterium tuberculosis infection can occur in all tissues of the body, pulmonary tuberculosis infection being the commonest, representing around $80 \%$ of all cases of tuberculosis [2]. Otorhinolaryngeal manifestations are uncommon, the commonest being lymphadenitis followed by laryngeal tuberculosis. Most physicians do not consider TB in their differentials when patients present with otorhinolaryngeal symptoms, thus, resulting in missed diagnosis and inappropriate treatment [2].

Tuberculosis runs an indolent course, as in this patient, whose symptoms persisted for 2 years, and was
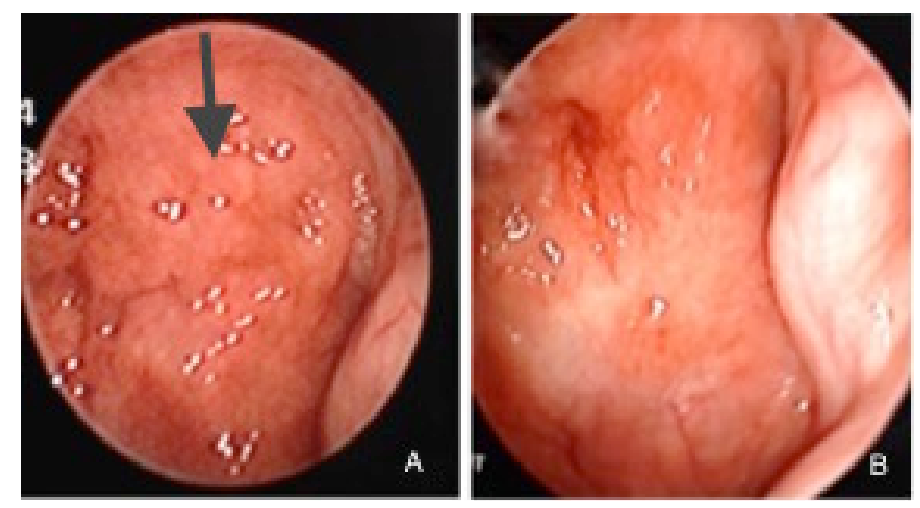

Figure 1. Rigid nasoendoscopy pre-treatment (A) and 5 months after intiation of treatment (B): left post nasal space mass in the former (A), and resolution in the latter (B).
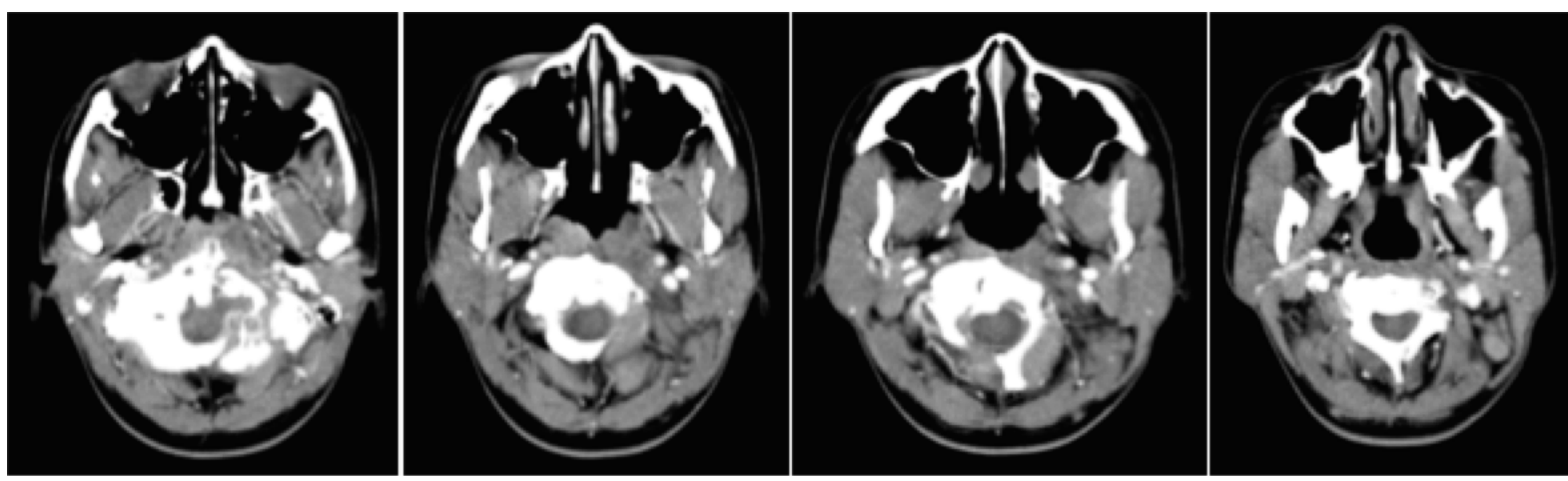

Figure 2. Axial CT scans showing bony destruction of lateral mass of C1 and C2, with asymmetry of the left fossa of Rosenmuller, and postnasal space bulge on the left. 


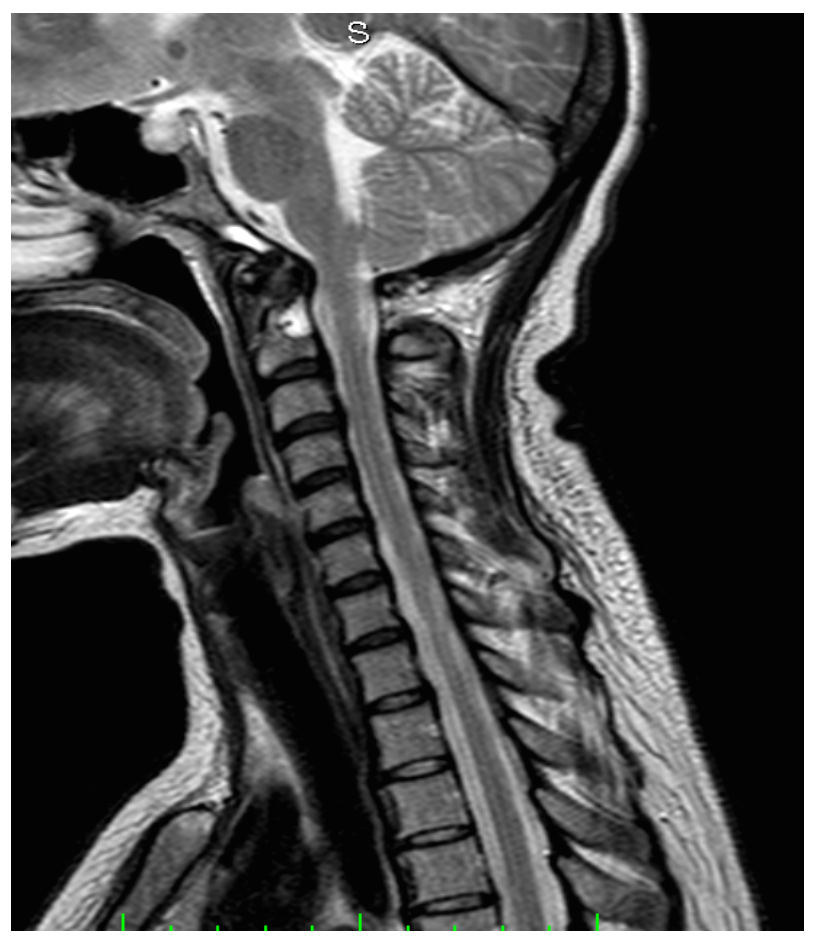

Figure 3. Sagittal MRI showing persistent but smaller C2 mass.

treated for migraine. Her presenting symptoms of neck pain and torticollis were also consistent with those case reports where patients present with TB in the ENT region with skull base involvement [3] [4].

The commonest vertebra to be affected by Mycobacterium tuberculosis in adults is the tenth thoracic vertebrae (T10), and the cervical spine is very much less affected, in which the prevalence is only $1.8 \%$ out of 165 cases of head and neck TB in the series by Prasad et al. [5], as isolated skull base TB is rare due to the fact that TB bone has a predilection for large and weight-bearing bones [3] [6]. In fact, Mancusi et al. reported that only $0.2 \%-1.3 \%$ of skeletal TB is localized in the skull, with involvement of the skull base occurring in only a few cases [6]. Thus this is an extremely rare form of extrapulmonary presentation of tuberculosis.

The pathogenesis of ENT TB is thought to be either from primary infection from Waldeyer's ring, from haematogenous spread from a TB focus in the lung or elsewhere, or direct inoculation from pulmonary TB. As the ear nose and throat are in close proximity with the skull base or cervical region, TB may be a manifestation of these different systems which are in close proximity [3]. It is the most probable that the patient has tuberculosis of the cervical spine leading to manifestation in the nasopharynx, given that the persistent lesion is in the C2 vertebrae.

This patient did not show evidence of pulmonary tuberculosis, but she had most probably already had TB during her pregnancy. The presentation of tuberculosis in pregnant women is similar to non-pregnant women, but it is more difficult to recognize the symptoms of malaise in pregnant women [7], as it is fairly usual in many, and loss of weight and appetite are symptoms difficult to pin point to tuberculosis especially when the patient is pregnant, unless she offers a history of contact with TB patients or is at high risk of exposure to tuberculosis.

Biopsy is essential for diagnosis and proper treatment initiation. The nasopharyngeal mass in this patient had facilitated in ease of biopsy. Although ENT TB demonstrated low yield many a time, as in the study by Michael et al., where only 5 out of 121 otorhinolaryngeal TB cases had histopathology and culture-proven TB [2], we managed to diagnose this case with a biopsy. She was initiated on standard anti-tuberculous therapy for the duration of 9 months and is still under follow up.

On follow-up, this patient demonstrated improvement in symptoms (resolution of headache, and reduction of neck stiffness), signs (resolution of nasopharyngeal mass), and imaging findings (smaller C2 mass) and is still on anti-tuberculosis therapy (aim of 9 months therapy) in accordance with national clinical practice guidelines. An imaging upon completion of treatment would be beneficial in assessing treatment outcome, and a bone biopsy would be essential if the lesion has not resolved, to rule out refractory cases or multi-drug resistant tuber- 
culosis.

\section{Conclusion}

A high index of suspicion is required when patients present with otorhinolaryngeal $\mathrm{TB}$, as it runs an indolent course, and its manifestations can vary depending on the anatomical site and the adjacent structures.

\section{Acknowledgements}

This report did not receive funding from any organization, thus there was no conflict of interest between the authors with any organization. Written consent has been obtained from the subject.

\section{References}

[1] MaHTAS (2012) Management of Tuberculosis. Malaysian Clinical Pracitce Guidelines. Malaysia Health Technology Assessment Section, Putrajaya. http://www.moh.gov.my/attachments/8612.pdf

[2] Michael, R.C. and Michael, J.S. (2011) Tuberculosis in Otorhinolaryngology: Clinical Presentation and Diagnostic Challenges. International Journal of Otolaryngology, 2011, Article ID: 686894. http://dx.doi.org/10.1155/2011/686894

[3] Richardus, R.A., et al. (2011) Two Immigrants with Tuberculosis of the Ear, Nose, and Throat Region with Skull Base and Cranial Nerve Involvement. Case Reports in Medicine, 2011, Article ID: 675807.

[4] Nalini, B. and Vinayak, S. (2006) Tuberculosis in Ear, Nose, and Throat Practice: Its Presentation and Diagnosis. American Journal of Otolaryngology, 27, 39-45. http://dx.doi.org/10.1016/j.amjoto.2005.07.005

[5] Prasad, K.C., Sreedharan, S., Chakravarthy, Y. and Prasad, S.C. (2007) Tuberculosis in the Head and Neck: Experience in India. The Journal of Laryngology \& Otology, 121, 979-985. http://dx.doi.org/10.1017/s0022215107006913

[6] Mancusi, G., Marks, B., Czerny, C., Thalhammer, F., Thurnher, D., Riedl, M., et al. (2005) Tubercular Osteomyelitis of the Clivus and the Nasopharynx. HNO, 53, 1081-1084. http://dx.doi.org/10.1007/s00106-005-1279-1

[7] Nguyen, H.T., Pandolfini, C., Chiodini, P. and Bonat, M. (2014) Tuberculosis Care for Pregnant Women: A Systematic Review. BMC Infectious Diseases, 14, 617. http://dx.doi.org/10.1186/s12879-014-0617-x 